\title{
Letters lost in space: hemispace dependent handwriting errors
}

\author{
Stephanie Ortigue, ${ }^{C A l, 2}$ Theodor Landis, ${ }^{3}$ Christoph M. Michel,' Jennifer Blanke, \\ Marie-Dominique Martory ${ }^{2}$ and Olaf Blanke $\mathrm{e}^{1,3,5}$
}

\begin{abstract}
'Functional Brain Mapping Laboratory; ${ }^{2}$ Neuropsychological Unit; ${ }^{3}$ Department of Neurology, University Hospital of Geneva, 24 rue Micheli du Crest, $\mathrm{CH}-$ I2II Geneva I4; ${ }^{4}$ Graduate Institute of International Studies, Geneva; ${ }^{5}$ Laboratory of Cognitive Neuroscience, Brain Mind Institute, Ecole Polytechnique Pédérale de Lausanne (EPFL), Lausanne, Switzerland

${ }^{\mathrm{CA}, \mathrm{I}}$ Corresponding Author and Address: stephanie.ortigue@hcuge.ch
\end{abstract}

Received I3 September 2004; accepted I8 September 2004

\begin{abstract}
Although handwriting is a daily life activity commonly attributed to the left hemisphere in the majority of right-handers, it is also known to require attentional and spatial mechanisms that rely on right hemispheric processing. The underlying spatial organization of handwriting in patients with right brain damage remains unresolved. Here we show in a patient with circumscribed right superior parietal damage that handwriting systematically depends on
\end{abstract}

the hand's position in space with respect to her body-midline. Most importantly, handwriting in contralesional space not only leads to spatial but also to language errors. This suggests that the right hemisphere's role in handwriting may surpass its generally assumed purely spatial contribution. We discuss our results in term of co-registration between both cerebral hemispheres in language processing. NeuroReport 15:2545-2548 (c) 2004 Lippincott Williams \& Wilkins.

Key words: Attention; Handwriting; Right parietal lobe; Spatial registration hypothesis

\section{INTRODUCTION}

Handwriting is a daily activity commonly attributed to the left hemisphere (especially to the left superior parietal lobe) in the majority of right-handers, as suggested by clinical studies in brain damaged patients [1], split brain patients [2], and healthy subjects [3-5]. Thus, theories of hemispheric specialization have strongly dissociated the left language dominant hemisphere from the right spatial dominant hemisphere. The left hemisphere has been demonstrated to mediate specific components of language, such as phonology, syntax, and semantic [1-4]. The right hemisphere on the other hand, has been demonstrated to mediate spatial attention according to different frames of reference and different compartments of space (for reviews see [6-8]). Accordingly it has been assumed that handwriting and spatial processing might be separated in our cognitive systems [9]. However, other data show that handwriting also requires some attentional and spatial mechanisms, which rely on right hemispheric processing (for reviews see $[2,10])$. For example, frequent pathology of handwriting after right parietal damage is spatial dysgraphia [11]. This is a deficit of handwriting characterized by a tendency to write predominantly on the right side of a sheet of paper, to produce wandering lines with overlapping words and letters, and to split words incorrectly. However, these dysgraphic errors are considered to be of purely spatial nature and have classically been opposed to agraphic errors (i.e. substitutions, transpositions, insertions of letters $[11,12])$, which are thought to be of language origin. In light of this, spatial dysgraphia has been suggested to highlight rather inter-hemispheric relationships than a pure contribution of the right hemisphere in handwriting [13]. Based on the spatial registration hypothesis (see below), one could suggest a strong interaction between handwriting and space, as it has been suggested for naming and reading (for reviews see $[9,14,15]$ ). The spatial registration hypothesis [14] suggests that every cognitive function, such as memory or language, is always maintained in registration with spatial systems. For example, Coslett and co-workers have demonstrated that actions of aphasic patients due to a lesion involving the left parietal cortex may improve their language abilities, within their ipsilesional hemispace such as naming and reading (for reviews see $[9,14,15])$. The spatial registration hypothesis suggests that spatial locations of stimuli are not only automatically encoded in a sensorimotor way as it can be highlighted by the well known Simon effect (shorter reaction times with the right hand for right-sided stimuli even when the spatial location of the stimulus is irrelevant to the task [16]), but that spatial locations also influence higher cognitive functions. From an anatomical point of view, these studies have demonstrated a crucial role of the parietal lobe in spatial registration and thus in cross-material (spatial-linguistic) integration (see [9] for review). Whereas patients with lesions in the left parietal lobe have been described to perform significantly worse on a variety of language tasks when they attend to right hemispace, the influence of right hemispheric lesions on handwriting remains unresolved. The role of the right hemisphere in writing is generally thought to be limited to spatial processing as evidenced by the presence of spatial (dysgraphic) errors due to right hemispheric brain damaged. These errors are generally assessed centrally according to patients' body midline $[4,5,17]$. Nevertheless, one could expect that right brain damaged patients might present language errors such as agraphic errors depending on the spatial compartment in which they act. 
Based on a current attentional theory [8] that suggests that the right hemisphere deals with both left and right visual field, while the left hemisphere only attends to the right visual field, one could indeed suppose that more severe handwriting errors will occur in the left hemispace than in the right hemispace after a right hemispheric damage. This spatial nature of handwriting following right hemispheric brain damage is also suggested by the fact that spatial dysgraphia is mainly observed in association with a spatial and attentional deficit, called hemineglect. Hemineglect is often exhibited after right brain damage and is characterized by a failure to attend to stimuli presented opposite to the side of brain damage [8]. Whereas hemineglect is usually investigated in both hemispaces separately [14,15], this has, to our knowledge, never been assessed in spatial dysgraphia [11]. We thus investigated this issue in a patient with spatial dysgraphia due to a right superior parietal lesion. Our hypothesis was that handwriting, like hemineglect, should be influenced systematically by the writing hand's position in space and might be more severely disrupted in the left hemispace.

\section{PATIENT AND METHODS}

Patient: The patient was a 63-year-old right-handed French-speaking woman who was initially referred to the Neurology Clinic for dressing and slight walking difficulties. More specifically, she reported difficulties in coordinating her left hand when dressing, accompanied by a feeling of numbness in the same hand, and a lack of control of her left arm. MRI showed circumscribed damage to the right superior parietal lobule (Fig. 1). Brain damage was due to bleeding from an arteriovenous malformation, which was operated 15 days after the onset of the symptomatology. The present evaluation was carried out prior to operation.

The neurological examination revealed mild left visual and tactile extinction as well as mild hypoesthesia, moderately impaired arm and digit position sense, and mildly impaired graphesthesia, all left-sided. Stereognosis and thermesthesia were preserved. There was no associated motor deficit and no signs of dressing apraxia or optic ataxia. The patient had suffered poliomyelitis at the age of 3 years, resulting in distal and proximal muscle loss of the left lower extremity.

A detailed neuropsychological examination, done immediately after admission and conducted in conformity with the Declaration of Helsinki, did not reveal any sign of dementia (preserved executive functions and memory abilities), aphasia (verbal fluency, denomination, reading, spelling, writing), or apraxia. There was also no evidence of callosal disconnection (i.e. no left anomia or apraxia and no right deficit in copying). However, deficits commonly observed after right brain damage, such as a moderate left perceptual hemineglect and a mild spatial dysgraphia, were found. There were also moderate difficulties in mental rotation and in judgment of line orientations.
Methods: Handwriting was tested by applying a $2 \times 2$ design in which we modified either the spatial position of the writing paper $(20 \mathrm{~cm}$ to the right or to the left from the body-midline) or the writing hand (left or right). The patient sat at a table in front of the experimenter such that her anterior chest wall was parallel to the edge of the table with her hands positioned symmetrically according to her body midline. The patient was not permitted to rotate or shift her body but was permitted to move her eyes and head.

In condition 1 (right handwriting in right hemispace), the sheet of paper was placed in the right hemispace from her midline and the patient had to write with her right hand. In condition 2 (right handwriting in left hemispace), the sheet of paper was placed in the left hemispace from her midline and she had to write again with her right hand. Conditions 3 (left handwriting in right hemispace) and 4 (left handwriting in left hemispace) were similar to the two previous ones except that the patient used her left hand. In the four experimental conditions, handwriting errors were evaluated by asking the patient to write to dictation: isolated French words $(n=7)$, alphabet letters $(n=26$ maximum $)$ and numbers ( $n=10$ maximum). All conditions were counterbalanced and conducted three times over multiple sessions. For clinical reasons, each condition resulted in a different number of total trials (left hand/left hemispace: 41; left hand/right hemispace: 42; right hand/left hemispace: 79; right hand/right hemispace: 91). Percentage of handwriting errors was calculated for each of the four experimental conditions. These error values were submitted to an ANOVA using the writing hand (left, right) and the writing hemispace (left, right) as within-subject factors.

\section{RESULTS}

Handwriting in both visual fields led to two qualitative different handwriting errors: some spatial (dysgraphic) errors, and some linguistic (agraphic) errors. This is astonishing because linguistic errors are generally attributed to left hemisphere damage. Dysgraphic errors were counted if the word was not written straight across an imaginary line and instead at least two letters drifted above or below this imaginary line. Agraphic errors were counted if there was at least a phonological paragraphia, a substitution, a transposition or a syntaxic error. Dysgraphic errors and agraphic errors were counted separately for each condition. For example, for right handwriting, left hemispace led to a phonological paragraphia (e.g., ciele for ciel (skye for sky), see Fig. 2; condition 2) and a persistent substitution in alphabet writing ( $Q$ for $K$ ). For left handwriting, left hemispace led to frequent feature errors, transpositions of numbers (e.g., E for 8; T for 7) and numerous reduplicative syntaxic errors (e.g., 209 for 29; 1034 for 134, see Fig. 2; condition 4). In addition, left handwriting in the left

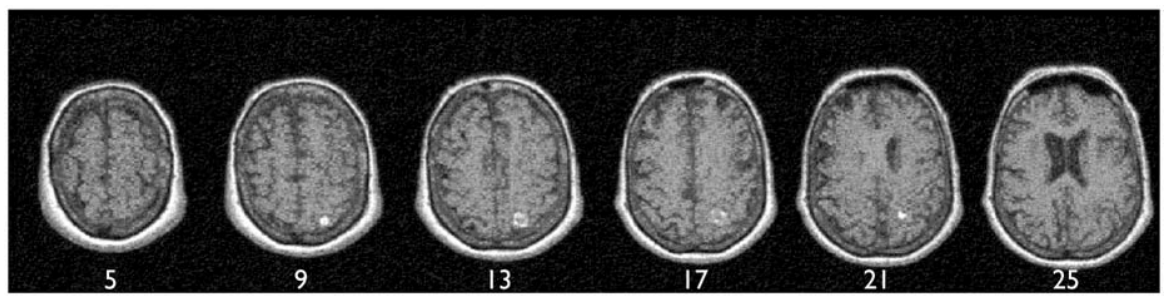

Fig. I. T2-weighted magnetic resonance imaging of bleeding in the right superior parietal lobule. Transverse slices (each $2 \mathrm{~mm}$ ) are shown to depict the lesion. 
hemispace also led to errors within letters (e.g., 3 instead of S), transpositions, substitutions and deletions of letters within words to dictation (e.g., cle for ciel (key for sky); see Fig. 2; condition 4). In the following three paragraphs, we will describe separately results of statistical analysis for all errors, dysgraphic errors, and finally agraphic errors.

Right and left handwriting were more impaired in left hemispace $(\mathrm{F}(1,5)=77.928, p<0.001$; left (conditions 2 and 4) > right (conditions 1 and 3): percentage of handwriting errors: $54 \%>34 \%)$. ANOVA of errors also revealed a significant main effect of the writing hand $(F(1,5)=35.602$, $p=0.002$; left (conditions 3 and 4) > right (conditions 1 and 2 ): percentage handwriting errors: $60 \%>28 \%$ ), indicating that the patient better performed with her right hand. No significant interaction was exhibited between these factors $(\mathrm{F}(1,5)=0.60425, p=0.4720)$.

For the dysgraphic errors, the analysis of patient's errors revealed a significant main effect of the writing hemispace $(\mathrm{F}(1,2)=110.16, p=0.009$; left $>$ right: percentage dysgraphic errors $73 \%>51 \%$ ), highlighting more frequent dysgraphic errors in left than in right hemispace. A significant main effect of writing hand was also found $(F(1,2)=26.783$, $p=0.035$; left $>$ right: percentage handwriting errors: $72 \%>51 \%)$. No interaction between the writing hand and the hemispace was observed $(F(1,2)=0.028, p=0.882)$.

The statistical analysis of agraphic errors revealed a significant main effect of the writing hemispace $(F(1,2)=$ 20.30, $p=0.046$; left (conditions 2 and 4) > right (conditions 1 and 3): percentage agraphic errors: $35 \%>17 \%$ ), indicating that agraphic errors were more prominent within the left hemispace. A significant main effect of the writing hand was also seen $(F(1,2)=65.11, p=0.015$; left (conditions 3 and 4) $>$ right (conditions 1 and 2): percentage agraphic errors: $47 \%>6 \%$ ). There was also a significant interaction $(F(1,2)=44.63, p=0.022)$, indicating that left handwriting was especially affected by agraphic errors in left hemispace.

\section{DISCUSSION}

While language is classically considered as a left hemispheric ability, the present observation suggests that handwriting after right brain damage may also be systematically influenced by the writing hand. In addition to these manual effects, the present data also exhibited a strong spatial effect on handwriting. Indeed, our patient exhibited more right and left handwriting errors in the left hemispace than in the right hemispace. This overall hemispace effect for the left side strongly reinforces the spatial registration hypothesis [14,15], which suggests that even cognitive function, which do not appear to have any intrinsic spatial organization such as language, are maintained in registration with spatial systems and are influenced by the hemispace in which the patient acts or directs his attention to.

Qualitatively, handwriting errors of our patient were of two kinds: spatial (dysgraphic) and linguistic (agraphic). Both types of handwriting error were differently modulated in space. First, our patient systematically produced more spatial handwriting errors (spatial dysgraphia) in the left hemispace of handwriting. Moreover, this spatial dysgraphia (tendency to write on the right side of a sheet of paper with some split words) was also enhanced with left handwriting. These results thus emphasize the admitted right hemispheric specialization for spatial abilities and their implication in spatial dysgraphia.

Second, in the left hemispace, handwriting errors were not only characterized by spatial errors, but also by linguistic errors (such as syntaxic errors, features' errors and transpositions), which are thought to be language specific. This suggests that after right brain damage, handwriting errors might not only be due to spatial deficits (such as spatial dysgraphia [11]), but also to language deficits (such as spatial agraphia). In light of this, we propose that language abilities, such as handwriting, might also depend upon where the hand is writing with respect to the body midline (egocentric frame of reference). Our neuropsychological results reinforce

\begin{tabular}{|c|c|c|c|c|c|}
\hline & & eft hand & & ight han & \\
\hline & $\begin{array}{l}\text { Left space } \\
\text { (Condition 4) }\end{array}$ & $\begin{array}{l}\text { Right space } \\
\text { (Condition 3) }\end{array}$ & $\begin{array}{l}\text { Left space } \\
\text { (Condition 2) }\end{array}$ & & $\begin{array}{l}\text { Right space } \\
\text { (Condition I) }\end{array}$ \\
\hline & - bC & "bol" Baff & $B O^{2}$ & "bol" & Bol \\
\hline$\frac{n}{0}$ & - $10 t 0$ & "moto" Molo & moto & "moto" & moto \\
\hline & - fire & "ciel" olal & $\diamond c^{e^{d e}}$ & "ciel" & cuel \\
\hline & - $\diamond 103 t$ & "134" 134 & $1^{34}$ & "134" & 134 \\
\hline$\frac{n}{0}$ & $\Leftrightarrow 400$ & "49" & 56 & "56" & 56 \\
\hline$\vec{z}$ & $\Leftrightarrow 209$ & "29" & $g 9$ & "29" & 29 \\
\hline & - $\circ y 1$ & $" 12 " / R$ 。 & 32 & "32" & 32 \\
\hline & 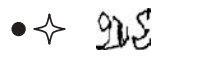 & "28" & & & \\
\hline
\end{tabular}

Fig. 2. Samples of the patient's handwriting in the four experimental conditions. Numbers and words written between quotation marks correspond to stimuli dictated by the experimenter. Condition I did not lead to any errors. Conditions 2 and 4 demonstrated an important spatial dysgraphia. Regarding language errors in contralesional (left) hemispace (conditions 2 and 4), black circles indicate features' errors and white circles correspond to transpositions (e.g. in condition 4/stimuli I2: the patient correctly began to write number I, but then hesitated where to put number 2 and finally wrote something in front of the I). White stars represent syntaxic errors for numbers or phonological errors for words. 
previous claims based on psychological studies that language and space strongly interact $[9,18]$.

From an anatomical point of view, our results support the assumed role of the right hemisphere and the superior parietal cortex in storing lexical templates that can be accessed by orthographic input for some language functions (for review see [19]). In line with this claim, some studies have demonstrated that aphasic patients with focal left hemisphere damage may have better performance in pronouncing and reading aloud emotional than neutral words $[17,20]$. Right hemisphere language mechanisms have also been assumed in the quality of implicit reading demonstrated by some patients who have lost the ability to explicitly read following left brain damage (for review see [21]).

The role of the right hemisphere in handwriting is still debated $[4,5,17]$. Whereas both language and space have been suggested to be mediated by widely distributed networks, involving the posterior temporo-parietal area, the dorso-lateral and medial prefrontal regions as well as the basal ganglia and the thalamus (for review see [22]), handwriting has been assumed to specifically involve the left superior parietal cortex $[3-5,11]$. However, in the present observation, we cannot accredit our patient's symptoms to this region or to a possible reorganisation of hemispheric language laterality from left to right because the patient was strongly right-handed for all skilled manual activities (Edinburgh Inventory). This was also confirmed by the absence of any family history of left-handedness and her left hemisphere dominance for language during a standard dichotic test.

In light of this, our data suggest a co-registration between the left and the right parietal lobes in handwriting. The present study thus suggests a crucial role of the right superior parietal lobe in handwriting not only in its ability to mediate the control of actions $[23,24]$ or spatial attention according to some egocentric frame of references [6,8,25], but also in the integration of linguistic-spatial components. We thus speculate that the increase of frequency of agraphic errors in contralesional space corroborates the implication of both superior parietal lobes in the registration and production of language according to the body midline. In light of this, it might be suggested that the superior parietal cortex, historically believed to be an association area, is also an area important not only for cross-modal (e.g. auditory-visual) integration (see [23] for review) but for cross-material (spatial-linguistic) integration as well.

\section{CONCLUSION}

In accordance with the spatial registration hypothesis, the present results show that even language specific material such as letters, words, and numbers, can be lost in space after right superior parietal lesion and lead to agraphia in contralesional space. This suggests that the right superior parietal lobe not only plays a role in mediating the control of actions in space $[23,24]$, but also in the control of language in space, reinforcing the idea that language and spatial cognition systematically interact [18]. Our results thus support the claim that spatial location might have a critical influence on the evolution of the primate nervous system and that higher cognitive abilities such as language, developed from brain structures initially mediating sensor motor interactions with the environment (see [15] for discussion).

\section{REFERENCES}

1. Déjérine J. Contribution a l'etude anatomo-pathologique et clinique des differentes varietes de cecite verbale. Mem Soc Biol 1892; 4:61-90.

2. Sperry RW, Gazzaniga MS and Bogen JE. Interhemispheric relationships: the neocortical commissures; syndromes of hemisphere deconnection. In: Vinken PJ and Bruyn GW (eds). Handbook of Clinical Neurology. Amsterdam: North Holland; 1969, pp. 273-290.

3. Cohen L, Dehaene S, Chochon F, Lehericy S and Naccache L. Language and calculation within the parietal lobe: a combined cognitive, anatomical and fMRI study. Neuropsychologia 2000; 38:1426-1440.

4. Menon V and Desmond JE. Left superior parietal cortex involvement in writing: integrating fMRI with lesion evidence. Cogn Brain Res 2001; 12:337-340.

5. Katanoda K, Yoshikawa K and Sugishita M. A functional MRI study on the neural substrates for writing. Hum Brain Mapp 2001; 13:34-42.

6. Husain $\mathrm{M}$ and Rorden C. Non-spatially lateralized mechanisms in hemispatial neglect. Nat Rev Neurosci 2003; 4:26-36.

7. Ortigue S, Viaud-Delmon I, Michel CM, Blanke O, Annoni JM, Pegna A et al. Pure imagery hemi-neglect of far space. Neurology 2003; 60:2000-2002.

8. Heilman KM, Watson RT, Bower D and Valenstein E. Right hemisphere dominance for attention. Rev Neurol 1983; 139:15-17.

9. Chatterjee A. Language and space: some interactions. Trends Cogn Sci 2001; 5:55-61.

10. Benson DF and Zaidel E. The Dual Brain: Hemisphere Specialization in Humans. New York: Guilford Press; 1985.

11. Lebrun Y. Disturbances of written language and associated abilities following damage to the right hemisphere. Appl Psycholing 1985; 6: 231-260.

12. Silveri MC. Peripheral aspects of writing can be differentially affected by sensorial and attentional defect: evidence from a patient with afferent dysgraphia and case dissociation. Cortex 1996; 32:155-172.

13. Heilman KM, Bowers D, Valenstein E and Watson RT. The right hemisphere: neuropsychological functions. J Neurosurg 1986; 64:693-704.

14. Coslett HB, Schwartz MF, Goldberg G, Haas D and Perkins J. Multimodal hemispatial deficits after left hemisphere stroke. A disorder of attention? Brain 1993; 116:527-554.

15. Coslett HB. Spatial influences on motor and language function. Neuropsychologia 1999; 37:695-706.

16. Simon JR. Reactions toward the source of stimulation. J Exp Psychol 1969; 81:174-176.

17. Landis T, Graves R and Goodglass H. Aphasic reading and writing: possible evidence for right hemisphere participation. Cortex 1982; 18: 105-112.

18. Majid A, Bowerman M, Kita S, Haun DBM and Levinson SC. Can language restructure cognition? The case of space. Trends Cogn Sci 2004; 8:109-114.

19. Larsen J, Baynes K and Swick D. Right hemisphere reading mechanisms in a global alexic patient. Neuropsychologia 2004; 42:1459-1476.

20. Landis T, Regard M, Graves R and Goodglass H. Semantic paralexia: a release of right hemispheric function from left hemispheric control. Neuropsychologia 1983; 21:359-364.

21. Coltheart M. Deep dyslexia is right-hemisphere reading. Brain Lang 2000; 71:299-309.

22. Mesulam MM. From sensation to cognition. Brain 1998; 121:1013-1052.

23. Andersen RA and Buneo CA. Intentional maps in posterior parietal cortex. Annu Rev Neurosci 2002; 25:189-220.

24. Rizzolatti G and Matelli M. Two different streams form the dorsal visual system: anatomy and functions. Exp Brain Res 2003; 153:146-157.

25. Vallar G, Lobel E, Galati G, Berthoz A, Pizzamiglio L and Le Bihan D. A fronto-parietal system for computing the egocentric spatial frame of reference in humans. Exp Brain Res 1999; 124:281-286. 